\title{
ANÁLISIS NUTRICIONAL Y NIVELES DE EXTRACCIÓN DEL MANGO CV. AZÚCAR EN EL DEPARTAMENTO DEL MAGDALENA, COLOMBIA
}

\author{
Marlon J. Yacomelo-Hernández¹, María M. Ramírez-Gómez ${ }^{2}$, Urley A. Pérez-Moncada², \\ Angela Arcila Cardona ${ }^{2}$, Francisco F. Carrascal Pérez ${ }^{1}$ y Elías Florez Cordero ${ }^{1}$
}

\begin{abstract}
RESUMEN
El mango cv. Azúcar es cultivado en el departamento del Magdalena, municipios de Santa Marta y Ciénaga principalmente, lugar donde expresa su mejor comportamiento agronómico y calidad de la fruta. El objetivo de este estudio fue determinar estándares generales de nutrientes a nivel foliar y cuantificar la extracción de nutrientes por la fruta durante el ciclo de desarrollo de la planta, además de realizar un diagnóstico nutricional mediante la implementación de dos métodos de diagnóstico, el sistema integrado de diagnóstico y recomendación (DRIS) y la desviación del óptimo porcentual (DOP). Se identificaron correlaciones significativas entre la productividad y las concentraciones de $\mathrm{P}, \mathrm{K}$ y $\mathrm{Mg}$ en tejido foliar, y se definieron las concentraciones para los árboles de mayor productividad. La mayor extracción por tonelada de fruta fue de potasio en las tres etapas evaluadas con 2,43, 2,24 y 1,67 $\mathrm{kg}$ del nutriente, seguido de nitrógeno con 1,87, 1,27 y 1,29 kg. $\mathrm{t}^{-1}$. De los microelementos la mayor extracción fue de hierro, seguido de boro, con 3,88 y 1,37 g. $\mathrm{t}^{-1}$, respectivamente. La extracción total de nutrientes por el mango cv. Azúcar, por tonelada cosechada fue cercana a $4 \mathrm{~kg}$ de nutrientes distribuidos en la siguiente manera: $43 \% \mathrm{~K}, 33,72 \% \mathrm{~N}, 8,38 \% \mathrm{Ca}, 6,56 \% \mathrm{P}, 4,69 \% \mathrm{Mg}$ y 2,61 $\% \mathrm{~S}$, con 1,04\% para el resto de los nutrientes. El orden de requerimiento nutricional a partir de la mayor deficiencia determinado por el método DRIS fue de $\mathrm{Mn}>\mathrm{Cu}>\mathrm{Fe}>\mathrm{N}>\mathrm{P}>\mathrm{S}$ y por el método $\mathrm{DOP} \mathrm{Cu}>\mathrm{N}>\mathrm{S}>\mathrm{Mg}>\mathrm{Fe}>\mathrm{P}$.

Palabras clave adicionales: Deficiencia y suficiencia nutricional, DOP, DRIS, extracción de nutrientes, fruticultura
\end{abstract}

\begin{abstract}
Nutritional analysis and extraction levels in mango 'Azucar' in Magdalena State, Colombia

The mango 'Azucar' is cultivated in the Department of Magdalena, Municipalities of Santa Marta and Ciénaga mainly, a place where it expresses its best agronomic behavior and the best fruit quality. The objective of this trial was to determine general standards of nutrients at the foliar level and to quantify the extraction of nutrients by the fruit during the development cycle of the plant, in addition to performing a nutritional diagnosis through the implementation of two diagnostic methods, the integrated diagnostic system and recommendation (DRIS) and the deviation from optimal percentage (DOP). Significant correlations were identified between productivity and the concentrations of $\mathrm{P}, \mathrm{K}$ and $\mathrm{Mg}$ in foliar tissue, and nutrient levels were defined for highly productive trees. The highest extraction per ton of fruit was for potassium in the three evaluated stages with $2.43,2.24$ and $1.67 \mathrm{~kg}$ of the nutrient, followed by nitrogen with $1.87,1.27$ and $1.29 \mathrm{~kg} \cdot \mathrm{t}^{-1}$. Among microelements, the highest extraction was for iron, followed by boron, with 3.88 and $1.37 \mathrm{~g} \cdot \mathrm{t}^{-1}$, respectively. The total extraction by mango 'Azucar' per ton of harvested fruit was close to $4 \mathrm{~kg}$ of nutrients distributed as follows: $43 \% \mathrm{~K}, 33.72 \% \mathrm{~N}, 8.38 \% \mathrm{Ca}, 6.56 \% \mathrm{P}, 4.69 \% \mathrm{Mg}$ and $2.61 \% \mathrm{~S}$, with $1.04 \%$ for the rest of nutrients. The order of nutritional requirement from the greatest deficiency determined by DRIS was $\mathrm{Mn}>\mathrm{Cu}>\mathrm{Fe}>\mathrm{N}>\mathrm{P}>\mathrm{S}$ and by the DOP method $\mathrm{Cu}>\mathrm{N}>\mathrm{S}>\mathrm{Mg}>\mathrm{Fe}>\mathrm{P}$.
\end{abstract}

Additional keywords: DOP, DRIS, fruticulture, nutritional deficiency and sufficiency, plant nutrient removal

\section{INTRODUCCIÓN}

El mango (Mangifera indica L.) se cultiva en muchas regiones tropicales y subtropicales en todo el mundo (Bekele et al., 2020). Ocupa el quinto lugar en consumo y es el tercer fruto tropical con mayor producción (Galán, 2013). En Colombia el área sembrada para el año 2019 fue de cercana a 33

Recibido: Diciembre 28, 2020

Aceptado: Abril 13, 2021

${ }^{1}$ Corporación Colombiana de Investigación Agropecuaria, Agrosavia. Centro de Investigación Caribia. Santa Marta, Colombia. e-mail: myacomelo@agrosavia.co (autor de correspondencia) fcarrascal@agrosavia.co; edflorez@agrosavia.co

${ }^{2}$ Corporación Colombiana de Investigación Agropecuaria, Agrosavia. Centro de Investigación Tibaitatá. e-mail: mmramirez@agrosavia.co; uperez@agrosavia.co; aarcila@ agrosavia.co 
mil hectáreas con una producción aproximada de 300 mil toneladas. Dentro de las variedades cultivadas se encuentra el mango cv. Azúcar que es un clon criollo, establecido principalmente en el departamento del Magdalena con un área plantada aproximada de 3 mil hectáreas y un rendimiento promedio de 13,87 toneladas por hectárea (Agronet, 2020).

Los bajos rendimientos reportados en Colombia en comparación con indicadores internacionales se deben probablemente entre otros aspectos al desconocimiento de requerimientos nutricionales de los diferentes cultivares establecidos, lo que hace que los productores sobre o subestimen las necesidades de la planta. Algunos autores concuerdan en que existe un vacío en el manejo de la fertilización del cultivo, aunque se tiene claro que el árbol en sus diferentes estados de desarrollo presenta distintos requerimientos nutricionales (Faria et al., 2016; Fu et al., 2019; Salehin et al., 2020).

La nutrición de las plantas contempla el uso y adaptación de diversas herramientas de diagnóstico, cuya interpretación constituye la base técnica para orientar la nutrición eficiente que se refleje en el aumento de los rendimientos. Un indicador utilizado en la formulación de planes de fertilización es la restitución de nutrientes, basada en la extracción realizada por el fruto, pues en ellos se encuentra una elevada proporción de los nutrientes presentes en las plantas (Obreza y Morgan, 2008; Li et al. 2017). De acuerdo con Nadir (1972), se estima que los nutrientes en el fruto representan un tercio o más de las necesidades totales de la planta.

Otro método consiste en la fertilización a partir del estado nutricional, siendo los análisis de tejido la forma directa de evaluar el estado nutricional de las plantas (Mourão, 2003; Zekri et al., 2005; Malavolta, 2006). El tejido de la hoja se considera la parte más importante de la planta donde ocurre la activación fisiológica y este tejido muestra fácilmente el trastorno nutricional. La interpretación de los contenidos de nutrientes en los análisis de las hojas para evaluar el estado nutricional de la planta puede hacerse mediante varios métodos, siendo el nivel crítico y rango de suficiencia los que se utilizan con mayor frecuencia (Beaufils, 1973; Puentes et al., 2016; Rodríguez et al., 2018).

Existen otros sistemas de diagnóstico, tales como el sistema integrado de diagnóstico y recomendación (DRIS) que señala el orden de limitación relativa de los elementos que son contemplados en el estudio nutricional (Beaufils, 1973). Tiene ventajas sobre el valor crítico y sobre los rangos de suficiencia para identificar las necesidades de nutrientes, elaborar programas de fertilización y estudiar la respuesta del rendimiento al aporte de fertilizantes (Beberly et al. 1984; Walworth et al., 1986). Este índice se basa en la desviación media de cada relación importante (N/P, $\mathrm{P} / \mathrm{N}$, entre otros) de su valor óptimo. Por lo tanto, el índice óptimo para cualquier elemento nutriente debe ser 0.00. Los índices negativos indican deficiencia y los índices positivos indican exceso (Mourão, 2004). Las normas DRIS se obtienen de un número grande de valores de concentración media nutrimental, de cultivos con alto rendimiento (Beaufils, 1973).

El método de la desviación del óptimo porcentual (DOP) es un procedimiento estadístico que compara la concentración del nutriente en la muestra respecto a su concentración óptima (Romero et al., 2014). Este índice de interpretación del análisis foliar permite, además del diagnóstico realista de una situación nutricional dada, conocer el orden de limitación tanto por exceso como por déficit de cada uno de los nutrientes considerados (Sandoval et al., 2019; Romero et al., 2014). Una situación nutrimental óptima, para cualquier elemento, es definida por el índice DOP igual a cero, dando el orden de limitación tanto por exceso como por déficit, de cada uno de los nutrientes bajo estudio (Montañés et al., 1993; Lucena, 1997).

El objetivo de este trabajo fue determinar estándares generales a nivel foliar y cuantificar la extracción de nutrientes por la fruta de mango cv. Azúcar durante su ciclo de desarrollo, además de realizar un diagnóstico nutricional mediante la implementación de dos métodos de diagnóstico.

\section{MATERIALES Y MÉTODOS}

Localización. El estudio se desarrolló en fincas productoras de mayor tecnificación en el departamento del Magdalena. Se seleccionaron seis fincas con suelos ubicados en el orden Entisoles, los cuales se caracterizan por ser suelos neutros con bajo contenido de materia orgánica (SSDS, 1993). Las características químicas específicas de los 
suelos bajo estudio (media \pm SD) indican $7,19 \pm 0,61$ de $\mathrm{pH}, 1,06 \pm 0,35 \%$ de materia orgánica, $0,39 \pm 0,27 \mathrm{dS} \cdot \mathrm{m}^{-1}$ de conductividad eléctrica y $12,77 \pm 4,14 \mathrm{cmol}_{\mathrm{c}} \cdot \mathrm{kg}^{-1}$ de capacidad de intercambio catiónica. El contenido detallado de nutrientes se presenta en el Cuadro 1. De acuerdo con estándares propuestos por Osorio (2014), las concentraciones de $\mathrm{P}, \mathrm{Ca}, \mathrm{Mg}, \mathrm{K}, \mathrm{Fe}, \mathrm{Cu}$ y $\mathrm{Zn}$ se encuentran en el rango de suficientes a altas, y las de $\mathrm{S}, \mathrm{Na}, \mathrm{Mn}$ y $\mathrm{B}$, en rango de bajas a muy bajas.

Concentración de nutrientes en tejido foliar, inflorescencia $y$ fruto en cuatro etapas de desarrollo. En cada finca se seleccionaron dos poblaciones de 12 árboles, la de mayor y menor productividad (menor productividad $<250 \mathrm{~kg}$ fruta árbol ${ }^{-1}$; mayor productividad $>250 \mathrm{~kg}$ fruta árbol ${ }^{-1}$ ). Los de mayor productividad correspondieron a árboles vigorosos y longevos, con mejor sanidad y calidad de fruta, según criterio del productor. El ciclo de cosecha se dividió en cuatro etapas; la etapa 1, plena floración; las etapas 2 y 3 correspondieron a los 35 y 70 días después de la floración (DDF), y la etapa 4 a los 97 DDF, tiempo en el cual los frutos se encuentran en óptimo estado de desarrollo para su comercialización y consumo.

Cuadro1. Concentración promedio, mínima y máxima de nutrientes en los suelos estudiados, cultivados con mango cv. Azúcar en el departamento del Magdalena

\begin{tabular}{cccc}
\hline $\begin{array}{c}\text { Macronutriente } \\
(\text { más Na) }\end{array}$ & $\begin{array}{c}\text { Concentración } \\
\left(\mathrm{mg} \cdot \mathrm{kg}^{-1}\right)\end{array}$ & Micronutriente & $\begin{array}{c}\text { Concentración } \\
\left(\mathrm{mg}^{-1} \mathrm{~kg}^{-1}\right)\end{array}$ \\
\hline $\mathrm{P}$ & $47,2(4,56-198)$ & $\mathrm{Fe}$ & $16,63(5,0-47,9)$ \\
$\mathrm{S}$ & $4,68(1,67-25,9)$ & $\mathrm{Mn}$ & $1,42(1,0-3,03)$ \\
& $\begin{array}{c}\text { Concentración } \\
\left(\mathrm{cmol}(+) \cdot \mathrm{kg}^{-1}\right)\end{array}$ & $\mathrm{Zn}$ & $1,39(1,0-3,34)$ \\
$\mathrm{K}$ & $0,46(0,10-1,11)$ & $\mathrm{Cu}$ & $1,31(1,0-3,55)$ \\
$\mathrm{Ca}$ & $9,55(4,78-17,8)$ & $\mathrm{B}$ & 0,38 \\
$\mathrm{Mg}$ & $2,38(1,21-5,63)$ & & \\
$\mathrm{Na}$ & $0,39(0,14-1,89)$ & & \\
\hline
\end{tabular}

Métodos de extracción (pH: VC-R-004 Versión 03, CE: NTC 5596:2008, MO: Walkey \& Black, P: VC-R-007 Versión 2, Ca, Mg, K y Na: ID-R-072 Versión 5, Fe: NTC 5526:2007, Al: KCl, B y S: Fosfato monobásico de calcio

Para cuantificar las concentraciones de nutrientes en tejido foliar se colectaron muestras foliares en cada población por finca. Para cada población (mayor o menor productividad) se seleccionaron 12 plantas que fueron agrupadas en tres lotes de cuatro plantas cada uno. A cada una de las plantas de cada lote se le extrajeron 40 hojas, correspondientes a 10 hojas por punto cardinal, para completar 120 hojas en el lote. Esa muestra compuesta representó la unidad experimental. Las hojas se tomaron de brotes no fructíferos, en ramas del tercio medio del árbol, con edad comprendida entre 6 y 7 meses, lo que suele corresponder a la hoja más baja de un brote nuevo, según lo reportado por Campos y Calderón (2016). Adicionalmente, se cosecharon muestras de frutos, compuestas por una mezcla de 20 frutos del tercio medio de la copa (cuatro por cada punto cardinal y cuatro más al azar) tomadas en cuatro plantas por población, para un total de cuatro muestras compuestas por población y finca. Adicionalmente, en la etapa 1 se colectaron 10 inflorescencias en cada árbol muestreado para cuantificar la concentración de nutrientes.

En todas las muestras de los distintos órganos se determinó la concentración de $\mathrm{P}, \mathrm{K}, \mathrm{Ca}, \mathrm{Mg}, \mathrm{S}, \mathrm{Fe}$, $\mathrm{Mn}, \mathrm{Zn}, \mathrm{Cu}$ y $\mathrm{B}$ utilizando digestión cerrada con ácido nítrico y peróxido de hidrógeno en relación $5: 2$, y la cuantificación de los elementos usando un espectrómetro de absorción atómica Agilent 280. La concentración de nitrógeno se obtuvo por el método de Kjeldahl, de acuerdo con la metodología EPA 351,3 y la concentración de boro mediante la Norma Técnica Colombiana 5404.

Los datos obtenidos de las concentraciones de nutrientes a nivel foliar fueron analizados utilizando el programa estadístico SAS versión Enterprise Guide 5,1 (Cary, NC, USA). Se realizaron análisis de varianza y pruebas de medias de rango múltiple de Tukey.

Generación de estándares generales de nutrientes en tejido foliar. Para la generación de 
estándares generales de nutrientes en tejido foliar se realizó un análisis de correlación y análisis de componentes principales (ACP) con la finalidad de determinar los nutrientes que presentan mayor asociación con la productividad. La generación de los estándares generales se efectuó a partir del cuartil más alto de los árboles que tuvieron mayor productividad, es decir, a partir de la población de mayor productividad se seleccionaron los árboles que dentro de esta población alcanzaron los mayores rendimientos (entre 80 y $95 \%$ del rendimiento relativo) y presentaron correlaciones significativas entre las concentraciones de nutrientes en tejido foliar y la productividad.

Extracción de nutrientes por la fruta. A partir del peso de materia fresca y seca de fruto, se calculó la cantidad de masa seca correspondiente a una tonelada de fruto fresco. Conociendo la concentración nutrimental de este material, se cuantificó la cantidad de nutrientes que se extraen para una tonelada de fruto. La determinación de nutrientes en los frutos se realizó utilizando la misma metodología usada en el análisis de tejido foliar.

Diagnóstico nutricional. Se realizó con base en las metodologías DRIS y DOP. Para el método DRIS se realizó el siguiente procedimiento:

1) Para cada población (alta y baja productividad) se generaron relaciones binarias del conjunto de nutrientes en ambas direcciones (N/P, P/N, N/K, $\mathrm{K} / \mathrm{N}$...) y así para todas las relaciones posibles (Cuadro 2).

Cuadro 2. Relaciones binarias posibles entre las concentraciones foliares del conjunto de nutrientes en cuatro etapas fenológicas del mango cv. Azúcar en el departamento del Magdalena

\begin{tabular}{lcccccccccc}
\hline \multicolumn{1}{c}{ Nutrientes } \\
\hline Norma & $\mathrm{Ca} / \mathrm{N}$ & $\mathrm{Ca} / \mathrm{Mg}$ & $\mathrm{Ca} / \mathrm{K}$ & $\mathrm{Ca} / \mathrm{S}$ & $\mathrm{P} / \mathrm{N}$ & $\mathrm{Ca} / \mathrm{N}$ & $\mathrm{Ca} / \mathrm{Mg}$ & $\mathrm{Ca} / \mathrm{P}$ & $\mathrm{Ca} / \mathrm{K}$ & $\mathrm{Ca} / \mathrm{S}$ \\
Media & 0,71 & 8,75 & 0,58 & 9,40 & 0,06 & 1,48 & 10,35 & 16,64 & 3,50 & 12,80 \\
$\mathrm{CV}$ & 28,83 & 45,36 & 24,92 & 17,28 & 15,36 & 26,66 & 35,67 & 30,27 & 34,99 & 15,49 \\
\hline Norma & $\mathrm{P} / \mathrm{Ca}$ & $\mathrm{P} / \mathrm{Mg}$ & $\mathrm{S} / \mathrm{P}$ & $\mathrm{S} / \mathrm{K}$ & $\mathrm{S} / \mathrm{Mg}$ & $\mathrm{N} / \mathrm{P}$ & $\mathrm{N} / \mathrm{K}$ & $\mathrm{N} / \mathrm{Mg}$ & $\mathrm{N} / \mathrm{S}$ & $\mathrm{S} / \mathrm{P}$ \\
Media & 0,09 & 0,73 & 1,21 & 0,06 & 0,90 & 11,20 & 2,37 & 6,90 & 8,92 & 1,28 \\
CV & 27,26 & 24,32 & 14,69 & 12,51 & 31,56 & 14,69 & 24,59 & 20,88 & 14,35 & 20,29 \\
\hline Norma & $\mathrm{K} / \mathrm{P}$ & $\mathrm{K} / \mathrm{Mg}$ & $\mathrm{N} / \mathrm{K}$ & $\mathrm{N} / \mathrm{Mg}$ & & $\mathrm{S} / \mathrm{K}$ & $\mathrm{S} / \mathrm{Mg}$ & $\mathrm{P} / \mathrm{K}$ & $\mathrm{P} / \mathrm{Mg}$ & $\mathrm{K} / \mathrm{Mg}$ \\
Media & 19,95 & 14,58 & 0,83 & 11,90 & & 0,27 & 0,79 & 0,21 & 0,62 & 3,05 \\
CV & 15,52 & 28,79 & 12,25 & 25,70 & & 27,31 & 26,47 & 13,76 & 23,69 & 28,95 \\
\hline \multicolumn{1}{c}{} & $\mathrm{Etapa} 3$ & & & & & $\mathrm{Etapa} 4$ & & \\
\hline Norma & $\mathrm{S} / \mathrm{N}$ & $\mathrm{S} / \mathrm{P}$ & $\mathrm{S} / \mathrm{K}$ & $\mathrm{S} / \mathrm{Ca}$ & $\mathrm{S} / \mathrm{Mg}$ & $\mathrm{N} / \mathrm{P}$ & $\mathrm{N} / \mathrm{K}$ & $\mathrm{N} / \mathrm{Ca}$ & $\mathrm{N} / \mathrm{Mg}$ & $\mathrm{N} / \mathrm{S}$ \\
Media & 0,11 & 2,16 & 0,29 & 0,10 & 0,92 & 13,89 & 2,67 & 0,85 & 7,82 & 9,60 \\
CV & 12,31 & 17,48 & 26,17 & 13,66 & 34,64 & 12,46 & 18,21 & 29,67 & 31,37 & 14,80 \\
\hline Norma & $\mathrm{N} / \mathrm{Mg}$ & $\mathrm{N} / \mathrm{K}$ & $\mathrm{N} / \mathrm{Ca}$ & $\mathrm{P} / \mathrm{N}$ & $\mathrm{P} / \mathrm{Ca}$ & $\mathrm{Ca} / \mathrm{Mg}$ & $\mathrm{Ca} / \mathrm{P}$ & $\mathrm{Ca} / \mathrm{K}$ & $\mathrm{Ca} / \mathrm{S}$ & $\mathrm{S} / \mathrm{P}$ \\
Media & 8,00 & 2,49 & 0,90 & 0,05 & 0,05 & 10,15 & 17,34 & 3,32 & 11,76 & 1,46 \\
CV & 26,49 & 19,42 & 20,97 & 9,14 & 26,26 & 45,75 & 23,69 & 25,70 & 15,89 & 11,79 \\
\hline Norma & $\mathrm{P} / \mathrm{Mg}$ & $\mathrm{K} / \mathrm{P}$ & $\mathrm{K} / \mathrm{Ca}$ & $\mathrm{K} / \mathrm{Mg}$ & $\mathrm{Mg} / \mathrm{Ca}$ & $\mathrm{S} / \mathrm{K}$ & $\mathrm{S} / \mathrm{Mg}$ & $\mathrm{P} / \mathrm{K}$ & $\mathrm{P} / \mathrm{Mg}$ & $\mathrm{K} / \mathrm{Mg}$ \\
Media & 0,42 & 7,81 & 0,38 & 3,29 & 0,12 & 0,28 & 0,84 & 0,19 & 0,57 & 3,03 \\
CV & 23,55 & 14,51 & 34,41 & 28,83 & 43,72 & 16,67 & 36,48 & 9,01 & 32,96 & 37,14 \\
\hline
\end{tabular}

Norma: indicador de referencia; CV: coeficiente de variación. Etapa 1: plena floración; Etapas 2, 3 y 4 corresponden a 35, 70 y 97 días después de la floración

2) Para cada relación se calculó el valor promedio y el coeficiente de variación, y se escogió solo una dirección del cociente $\mathrm{A} / \mathrm{B}$ o $\mathrm{B} / \mathrm{A}$, teniendo en cuenta que la dirección seleccionada es aquella para la cual su cociente de varianzas entre alta y baja producción presenta el mayor valor. Una vez 
definida la dirección de la relación, se tomó su valor medio en la población de alta producción como valor de referencia y este valor es el que hizo parte de la norma

3) Se calcularon las funciones que comparan los datos analíticos con los de la norma, mediante las ecuaciones 1 ó 2 según el caso.

$$
\begin{aligned}
& F\left(\frac{A}{B}\right)=\left[1-\frac{a b}{A B}\right] \times \frac{1000}{C V \%} \text { Ecuación (1) } \\
& F\left(\frac{A}{B}\right)=\left[\frac{A B}{a b}-1\right] \times \frac{1000}{C V \%} \text { Ecuación (2) }
\end{aligned}
$$

donde $\mathrm{ab}$ corresponde a la concentración de referencia de los nutrientes y $\mathrm{AB}$ al resultado del análisis foliar.

La ecuación 1 se implementó cuando ab fue mayor que $\mathrm{AB}$ y la ecuación 2 cuando $\mathrm{AB}$ fue mayor o igual que ab.

4) Se calcularon los índices específicos de cada nutriente a partir de la función de las relaciones en las que intervinieron, tomando en cuenta que si la relación es directa se le mantiene el signo (+), pero si es inversa se le cambia de signo (-). De esta forma, el índice puede tener valores positivos o negativos y representan el promedio aritmético de funciones en las que está involucrado el nutriente. Cuando el resultado es negativo significa deficiencia y cuando es positivo indica exceso, como lo señala Beaufils (1973):

$$
\text { DRIS Index } A=\frac{\sum f\left(\frac{A}{B}\right)-f\left(\frac{B}{A}\right)}{n} \quad \text { Ecuación (3) }
$$

Finalmente, para interpretar los resultados mediante el método DOP, los índices de cada nutriente se calcularon a partir de valores de las muestras mediante la ecuación 4:

$$
I A=\frac{A-a}{A} x 100 \quad \text { Ecuación (4) }
$$

donde: $\mathrm{IA}=$ índice del nutriente, $\mathrm{A}=$ concentración de la muestra, $\mathrm{a}=$ valor de la norma.

La comparación de la funcionalidad de los métodos de diagnóstico DOP y DRIS se efectuó sobre los resultados del análisis foliar realizado a una población de árboles de las fincas seleccionadas.

Una vez calculados cada uno de los índices de los nutrientes, se determinó el Índice de Balance Nutricional (IBN), que es la suma de los valores absolutos de cada uno de los índices para cada elemento mineral, mediante la siguiente fórmula:

$$
\begin{aligned}
& I B N=|I(N)|+|I(P)|+|I(K)|+|I(C a)|+|I(M g)|+|I(S)| \\
& \text { Se consideró que cuanto mayor fue el IBN, } \\
& \text { mayor era el desequilibrio nutricional (Beaufils, }
\end{aligned}
$$

1973; Mourão, 2003).

\section{RESULTADOS Y DISCUSIÓN}

Las concentraciones de los nutrientes en los árboles de mango de cv. Azúcar variaron entre etapa fenológica para las hoajs y frutos de la planta (Cuadro 3).

En la etapa 1, los nutrientes que presentaron mayor variación en su concentración fueron $\mathrm{Cu}$, Fe y $\mathrm{Mn}$ en la inflorescencia, y $\mathrm{Cu}, \mathrm{Zn}$ y $\mathrm{K}$ en las hojas. En la etapa 2, $\mathrm{Mn}, \mathrm{Cu}$ y $\mathrm{Fe}$ en frutos, y $\mathrm{Cu}$, $\mathrm{Na}$ y $\mathrm{B}$ en hojas. En la etapa $3, \mathrm{Cu}, \mathrm{Na}$ y $\mathrm{Fe}$ en frutos, $\mathrm{Cu}, \mathrm{B}$ y $\mathrm{Mn}$ en hojas.

Finalmente, en la etapa 4, una vez que la planta ha completado la producción de frutos y éstos se encuentran óptimos para cosecha, la variación de las concentraciones de los nutrientes disminuyeron respecto a las tres etapas anteriores, siendo el nutriente $\mathrm{Cu}$, tanto en fruto como hoja, el que presentó mayor coeficiente de variación $(\mathrm{Cu}, \mathrm{CV}$ fruto $=56,57 \%$ y $\mathrm{CV}$ hoja $=86,76 \%)$. Las concentraciones variaron significativamente entre las etapas fenológicas, a excepción de las concentraciones de N, Na y B en hojas y Fe en frutos. En la etapa 1 las mayores concentraciones de nutrientes en hojas e inflorescencias fueron de $\mathrm{N}$ y $\mathrm{K}$; en las etapas 2, 3 y 4 las mayores concentraciones en hojas fueron de $\mathrm{Ca}$, seguido de $\mathrm{N}$, mientras que en fruto las mayores concentraciones fueron de $\mathrm{K}$, seguido de $\mathrm{N}$.

$\mathrm{La}$ variación de las concentraciones de nutrientes en tejido foliar por etapa fenológica ha sido reportada también para otros cultivares de mango. Por ejemplo, Faria et al. (2016) publicaron que en mango Tommy Atkins, la concentración foliar del conjunto de nutrientes varió según las etapas y ciclos de desarrollo de manera independiente, donde los nutrientes con menor movilidad en la planta ( $\mathrm{Ca}, \mathrm{B}, \mathrm{Fe}$ y $\mathrm{Mn}$ ), aumentaron en las hojas desde la floración hasta la fructificación, mientras que los de movilidad alta $(\mathrm{N}, \mathrm{P}, \mathrm{K}$ y $\mathrm{Mg}$ ) disminuyeron. Teixeira et al. (2019), en mango "Kent" en Brasil, también reportan variaciones en las concentraciones de nutrientes desde la etapa de prefloración hasta la floración. Medeiros et al. (2004) informaron que el $\mathrm{K}$ foliar fue alto en floración en comparación con otras etapas fenológicas del cultivo del mango y disminuyó desde la floración hasta la fructificación lo cual concuerda con los resultados encontrados en 
nuestra investigación. El potasio mostró una gran removilización desde las hojas hacia los frutos, lo que demuestra la importancia de generar estándares nutricionales para los diferentes cultivares establecidos en las regiones productoras, pues un estado nutricional adecuado es fundamental para el proceso de fructificación de los árboles de mango (Teixeira et al. 2019).

Cuadro 3. Concentración de nutrientes (\%) en el tejido de diferentes órganos del mango cv. Azúcar durante cuatro etapas fenológicas en el departamento del Magdalena

\begin{tabular}{|c|c|c|c|c|c|c|}
\hline \multirow{5}{*}{ Etapa } & $\mathrm{N}$ & $\mathrm{P}$ & $\mathrm{K}$ & $\mathrm{Ca}$ & $\mathrm{Mg}$ & $\mathrm{Na}$ \\
\hline & --------------.. & -------------------. & -------- $\%$ & ------------------. & -----------------. & --------------. \\
\hline & \multicolumn{6}{|c|}{ Inflorescencia (en paréntesis, el CV) } \\
\hline & $1,56(8,07)$ & $0,16(16,42)$ & $0,82(9,20)$ & $0,34(34,41)$ & $0,12(19,43)$ & $0,03(0,01)$ \\
\hline & \multicolumn{6}{|c|}{ Hojas } \\
\hline 1 & $1,49 \mathrm{a}$ & $0,10 \mathrm{c}$ & $1,39 \mathrm{a}$ & $1,13 \mathrm{~b}$ & $0,15 \mathrm{~b}$ & $0,03 \mathrm{a}$ \\
\hline 2 & $1,51 \mathrm{a}$ & $0,14 \mathrm{a}$ & $0,74 \mathrm{~b}$ & $2,23 \mathrm{a}$ & $0,23 \mathrm{a}$ & $0,04 \mathrm{a}$ \\
\hline 3 & $1,50 \mathrm{a}$ & $0,08 \mathrm{~d}$ & $0,66 \mathrm{~b}$ & $1,97 \mathrm{a}$ & $0,21 \mathrm{a}$ & $0,03 \mathrm{a}$ \\
\hline \multirow[t]{2}{*}{4} & $1,48 \mathrm{a}$ & $0,11 \mathrm{~b}$ & $0,63 \mathrm{~b}$ & $2,08 \mathrm{a}$ & $0,21 \mathrm{a}$ & $0,02 \mathrm{a}$ \\
\hline & \multicolumn{6}{|c|}{ Fruto } \\
\hline 2 & $0,77 \mathrm{a}$ & $0,09 \mathrm{c}$ & $1,00 \mathrm{a}$ & $0,22 \mathrm{a}$ & $0,10 \mathrm{a}$ & $0,03 \mathrm{a}$ \\
\hline 3 & $0,52 \mathrm{~b}$ & $0,07 \mathrm{~b}$ & $0,92 \mathrm{a}$ & $0,12 \mathrm{~b}$ & $0,07 \mathrm{~b}$ & $0,01 \mathrm{~b}$ \\
\hline 4 & $0,53 \mathrm{~b}$ & $0,10 \mathrm{a}$ & $0,69 \mathrm{~b}$ & $0,13 \mathrm{~b}$ & $0,07 \mathrm{~b}$ & $0,01 \mathrm{~b}$ \\
\hline \multirow{5}{*}{ Etapa } & $\mathrm{S}$ & $\mathrm{Fe}$ & $\mathrm{Cu}$ & $\mathrm{Mn}$ & $\mathrm{Zn}$ & B \\
\hline & ------- \% ------- & |----------------- & -------------- & $\mathrm{ng} \cdot \mathrm{kg}^{-1} \quad$------ & 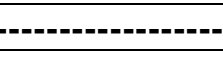 & $--------\cdot$ \\
\hline & \multicolumn{6}{|c|}{ Inflorescencia (en paréntesis, el CV) } \\
\hline & $0,10(11,55)$ & $110,75(59,47)$ & $4,47(63,26)$ & $39,77(48,40)$ & $17,10(22,37)$ & $16,90(13,67)$ \\
\hline & \multicolumn{6}{|c|}{ Hojas } \\
\hline 1 & $0,12 \mathrm{c}$ & $79,25 \mathrm{~b}$ & $61,82 \mathrm{a}$ & $129,57 \mathrm{~b}$ & $21,43 \mathrm{a}$ & $51,81 \mathrm{a}$ \\
\hline 2 & $0,18 \mathrm{a}$ & $48,19 \mathrm{c}$ & $10,18 \mathrm{~b}$ & $171,29 \mathrm{ab}$ & $11,69 \mathrm{~b}$ & $55,27 \mathrm{a}$ \\
\hline 3 & $0,18 \mathrm{a}$ & $116,93 \mathrm{a}$ & $6,93 \mathrm{~b}$ & $216,18 \mathrm{a}$ & $10,89 \mathrm{~b}$ & $52,26 \mathrm{a}$ \\
\hline \multirow[t]{2}{*}{4} & $0,16 b$ & $55,31 \mathrm{c}$ & $15,67 \mathrm{~b}$ & $174,81 \mathrm{ab}$ & $11,46 \mathrm{~b}$ & $55,18 \mathrm{a}$ \\
\hline & \multicolumn{6}{|c|}{ Fruto } \\
\hline 2 & $0,06 \mathrm{a}$ & $15,40 \mathrm{a}$ & $2,86 \mathrm{a}$ & $7,95 \mathrm{a}$ & $6,12 \mathrm{a}$ & $10,07 \mathrm{a}$ \\
\hline 3 & $0,04 \mathrm{~b}$ & $19,87 \mathrm{a}$ & $5,72 \mathrm{a}$ & $6,33 a b$ & $4,70 \mathrm{~b}$ & $6,27 \mathrm{~b}$ \\
\hline 4 & $0,04 \mathrm{~b}$ & $15,99 \mathrm{a}$ & $3,19 \mathrm{a}$ & $5,08 \mathrm{~b}$ & $4,64 \mathrm{~b}$ & $5,65 \mathrm{~b}$ \\
\hline
\end{tabular}

CV: coeficiente de variación (\%). Letras distintas entre filas en hojas y frutos muestran diferencias significativas según la prueba de Tukey $(P \leq 0,05)$

Población de mayor productividad vs. población de menor productividad. $\mathrm{Al}$ comparar las concentraciones de nutrientes en tejido foliar entre las dos poblaciones de árboles evaluadas, sólo se encontraron diferencias significativas $(P \leq 0,05)$ en las etapas 2 y 4 . En la etapa 2 se observaron diferencias en las concentraciones de $\mathrm{K}$, siendo mayor en árboles que expresaron mayor productividad $(0,82 \%$ vs. $0,67 \%)$. En la etapa 4 se observaron diferencias en las concentraciones de $\mathrm{P}$ $(0,13 \%$ vs. $0,11 \%), \mathrm{K}(69 \%$ vs. $58 \%)$ y $\mathrm{Mg}(19 \%$ vs. $24 \%$ ), siendo mayor los niveles de $\mathrm{P}$ y $\mathrm{K}$ en los árboles que presentaron mayor productividad.

El Cuadro 4 presenta las concentraciones obtenidas en los árboles de mayor productividad. El análisis de correlación realizado entre el conjunto de nutrientes en la etapa 4, es decir, la fase en la que se presentaron mayores diferencias en las concentraciones de nutrientes en tejido foliar entre las dos poblaciones, mostró que los nutrientes con el mayor número de correlaciones significativas fueron $\mathrm{P}$ y $\mathrm{Mg}$, de la siguiente manera: $\mathrm{P}$ vs. $\mathrm{K}, \mathrm{Mg}$, S, Fe y B; $\mathrm{Mg}$ vs. $\mathrm{P}, \mathrm{Fe}, \mathrm{Cu}$, $\mathrm{Mn}, \mathrm{Zn}$ y B (Cuadro 5). Shaban et al. (2019) reportaron la interacción que existe entre los nutrientes, señalando que el aumento en la tasa de aplicación de $\mathrm{B}$, incrementó las concentraciones de $\mathrm{N}, \mathrm{P}$ y $\mathrm{K}$ en hojas, lo que indica que existe una 
interacción sinergística entre el boro y el N-P-K foliar, lo que mejora su absorción y translocación. Ranade (2011) definió el sinergismo como un efecto positivo entre los nutrientes, en el que dos o más elementos funcionan en conjunto para crear un estado fisiológico mejorado general en la planta.

Cuadro 4. Concentración de nutrientes en la población de árboles de mango cv. Azúcar de mayor productividad durante cuatro etapas fenológicas en el departamento del Magdalena

\begin{tabular}{|c|c|c|c|c|c|c|c|c|c|c|c|c|}
\hline Etapa & $\mathrm{N}$ & $\mathrm{P}$ & K & $\mathrm{Ca}$ & $\mathrm{Mg}$ & $\mathrm{Na}$ & $S$ & $\mathrm{Fe}$ & $\mathrm{Cu}$ & $\mathrm{Mn}$ & $\mathrm{Zn}$ & B \\
\hline & & & & $(\%)$ & & & & \multicolumn{5}{|c|}{$\left(\mathrm{mg} \cdot \mathrm{kg}^{-1}\right)$} \\
\hline 1 & 1,48 & 0,10 & 1,33 & 1,13 & 0,13 & 0,04 & 0,12 & 67,00 & 50,62 & 129,9 & 19,43 & 50,58 \\
\hline 2 & 1,54 & 0,15 & 0,82 & 2,24 & 0,22 & 0,06 & 0,18 & 43,15 & 13,65 & 159,4 & 11,51 & 42,48 \\
\hline 3 & 1,51 & 0,08 & 0,71 & 1,94 & 0,20 & 0,02 & 0,18 & 106,4 & 9,59 & 216,2 & 11,21 & 50,23 \\
\hline 4 & 1,47 & 0,13 & 0,69 & 2,10 & 0,19 & 0,02 & 0,16 & 52,71 & 19,63 & 169,2 & 11,50 & 49,24 \\
\hline
\end{tabular}

Cuadro 5. Análisis de correlación entre las distintas concentraciones foliares de nutrientes y el rendimiento del mango cv. Azúcar en el departamento del Magdalena

\begin{tabular}{|c|c|c|c|c|c|c|c|c|c|c|c|c|}
\hline & $\mathrm{P}$ & K & $\mathrm{Ca}$ & $\mathrm{Mg}$ & $\mathrm{Na}$ & $S$ & $\mathrm{Fe}$ & $\mathrm{Cu}$ & $\mathrm{Mn}$ & $\mathrm{Zn}$ & B & Rendimiento \\
\hline $\mathrm{N}$ & $0,40 *$ & $-0,07$ & $-0,25$ & $-0,15$ & $-0,16$ & 0,34 & $-0,19$ & $-0,21$ & $-0,06$ & $-0,01$ & $-0,54 *$ & $-0,08$ \\
\hline $\mathrm{P}$ & & $0,64 *$ & 0,28 & $-0,44^{*}$ & $-0,33$ & $0,53^{*}$ & $-0,43^{*}$ & $-0,08$ & 0,12 & 0,04 & $-0,54^{*}$ & $0,51^{*}$ \\
\hline $\mathrm{K}$ & & & 0,2 & $-0,37$ & $-0,34$ & 0,12 & $-0,28$ & $-0,03$ & 0,18 & 0,07 & $-0,05$ & $0,80 *$ \\
\hline $\mathrm{Ca}$ & & & & $-0,37$ & 0,28 & $0,68^{*}$ & $-0,32$ & 0,26 & $0,52^{*}$ & 0,15 & $-0,03$ & 0,14 \\
\hline $\mathrm{Mg}$ & & & & & 0,16 & $-0,39$ & $0,65^{*}$ & $-0,43^{*}$ & $-0,48^{*}$ & $-0,44^{*}$ & $0,54 *$ & $-0,48^{*}$ \\
\hline $\mathrm{Na}$ & & & & & & 0,06 & 0,35 & 0,16 & $-0,08$ & 0,14 & 0,36 & $-0,38$ \\
\hline$S$ & & & & & & & $-0,54^{*}$ & 0,23 & $0,41^{*}$ & 0,04 & $-0,56^{*}$ & 0,02 \\
\hline $\mathrm{Fe}$ & & & & & & & & $-0,25$ & $-0,34$ & $-0,43^{*}$ & $0,57 *$ & $-0,23$ \\
\hline $\mathrm{Cu}$ & & & & & & & & & 0,17 & 0,08 & $-0,22$ & 0,11 \\
\hline $\mathrm{Mn}$ & & & & & & & & & & 0,3 & $-0,25$ & 0,11 \\
\hline $\mathrm{Zn}$ & & & & & & & & & & & 0,04 & 0,01 \\
\hline B & & & & & & & & & & & & $-0,06$ \\
\hline
\end{tabular}

*Indican correlaciones significativas al $5 \%$

En cuanto al rendimiento, se presentó una correlación significativa positiva con las concentraciones de $\mathrm{P}, \mathrm{K}$, y negativa con Mg. Esto concuerda con lo reportado por Dar et al. (2015) quienes observaron una relación significativa y positiva del fósforo $\mathrm{y}$ potasio foliar con el rendimiento mientras que el $\mathrm{Mg}$ mostró una relación significativa y negativa con el azúcar total en la fruta de pera. A su vez Bibi et al. (2019) encontraron un efecto positivo de aplicaciones foliares de potasio sobre la calidad y el rendimiento de la fruta de mango cv. SB Chaunsa.

Lo anterior podría atribuirse a que el fósforo cumple un papel fundamental como componente esencial de la célula, en el metabolismo de las plantas y en la transferencia de energía, y que el K está involucrado en las características relacionadas con la calidad de la fruta, por lo algunos autores lo han denominado elemento de la calidad (Ahmad et al., 2018). El K es crucial para muchas reacciones bioquímicas que son esenciales para la activación de enzimas y los procesos fisiológicos en la célula (Anees et al., 2016). El tamaño, la apariencia, el color, los sólidos solubles, la acidez, el contenido de vitaminas, el sabor y la vida útil de la fruta están significativamente influenciados por un suministro adecuado de K (Tohidloo et al., 2018).

Extracción de nutrientes por la fruta. El proceso de formación del fruto de mango cv. Azúcar demoró aproximadamente 97 días, tiempo en el 
cual se encontraba en óptimas condiciones de calidad para ser cosechado. Su mayor crecimiento se expresó hasta los 60 días, alcanzando hasta $80 \%$ de su máximo crecimiento, momento en el que inicia su proceso de maduración.

Las cantidades de nutrientes extraídos y translocados al fruto de mango cv. Azúcar se presentan en el Cuadro 6. La mayor extracción por tonelada de fruta fue de $\mathrm{K}$ en las tres etapas evaluadas con 2,43, 2,24 y 1,67 kg del nutriente por tonelada de fruta cosechada, seguido de $\mathrm{N}$ con 1,87 , 1,27 y $1,29 \mathrm{~kg}$. Los microelementos también tuvieron un papel importante en la formación de los frutos, pues son necesarios para diferentes procesos fisiológicos y metabólicos, y su deficiencia afecta una serie de procesos que incluyen el crecimiento, la productividad y la calidad de las plantas (Souri y Aslani, 2018a; Souri y Bakhtiarizade, 2019). Para mango cv. Azúcar la mayor extracción fue de Fe, seguido de B.

Cuadro 6. Extracción de nutrientes por el fruto del mango cv. Azúcar en el departamento del Magdalena

\begin{tabular}{|c|c|c|c|c|c|c|c|c|c|c|c|c|}
\hline apa & $\mathrm{N}$ & $\mathrm{P}$ & K & $\mathrm{Ca}$ & $\mathrm{Mg}$ & $\mathrm{Na}$ & S & $\mathrm{Fe}$ & $\mathrm{Cu}$ & $\mathrm{Mn}$ & $\mathrm{Zn}$ & B \\
\hline & \multicolumn{12}{|c|}{ 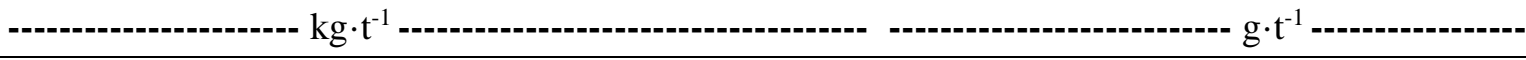 } \\
\hline 2 & & & & & & & & & & 1 , & 1,4 & $2,44 \mathrm{a}$ \\
\hline 3 & $1,27 \mathrm{~b}$ & $0,17 \mathrm{c}$ & 2 , & $0,30 \mathrm{~b}$ & 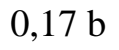 & 0, & b & , & $\mathrm{a}$ & 1,0 & 1,1 & b \\
\hline 4 & $29 \mathrm{~b}$ & $25 \mathrm{a}$ & $1,67 \mathrm{~b}$ & $0,32 \mathrm{~b}$ & $0,18 \mathrm{~b}$ & $0,01 \mathrm{~b}$ & $0,10 \mathrm{~b}$ & $3,88 \mathrm{a}$ & $0,77 \mathrm{a}$ & $1,23 \mathrm{~b}$ & $1,12 \mathrm{~b}$ & $1,37 \mathrm{~b}$ \\
\hline
\end{tabular}

Letras distintas entre filas muestran diferencias significativas según la prueba de Tukey $(P \leq 0,05)$

La mayor extracción de nutrientes se presenta en los primeros 40 días de formación para la mayoría de los nutrientes, lo cual difiere de algunos reportes de otros cultivares. Fallas et al. (2010) reportaron que en las primeras etapas el fruto en mango Tommy Atkins y Keith absorbe pequeñas cantidades de N, P y K y el incremento en la absorción de estos nutrientes se inicia a partir del período entre los 61 y 79 días después de floración; para esta fase el fruto extrajo más del doble de las cantidades absorbidas en las dos evaluaciones anteriores.

Una vez completado el ciclo fenológico, la extracción de nutrientes por tonelada de fruta cosechada fue de 3,83 $\mathrm{kg}$ de nutrientes (Cuadro 6; sumatoria de las extracciones en la etapa 4) repartidos de la siguiente manera: $43 \% \mathrm{~K}, 33,72$ $\% \mathrm{~N}, 8,38 \% \mathrm{Ca}, 6,56 \% \mathrm{P}, 4,69 \% \mathrm{Mg}, 2,61 \% \mathrm{~S}$ y el resto de los nutrientes las concentraciones son menores de $5 \%$. En Colombia no se dispone de referencias o antecedentes de la extracción de nutrientes en mango cv. Azúcar ni para otros cultivares; sin embargo, existen algunas referencias internacionales, como la de Mellado et al. (2020) en México, quienes encontraron que la extracción total de nutrientes por la fruta de mango no varió significativamente entre los cultivares Ataulfo, Tommy Atkins y Kent a excepción de las concentraciones de $\mathrm{K}, \mathrm{Mg}$ y $\mathrm{B}$. En cuanto a $\mathrm{K}$ y $\mathrm{Mg}$, se extrajeron en mayores cantidades en Ataulfo dependiendo de la región donde se cultiva. En el caso de B, fue extraído en mayores cantidades por el fruto de Kent, también dependiendo de la región cultivada. Cruz et al. (2014) en mango Ataulfo y Fallas et al. (2010) determinaron la extracción por cada kilogramo de fruta fresca de Tommy Atkins y Keith, respectivamente, y hallaron resultados, que a pesar de referirse a otros cultivares de mango, concuerdan con nuestro estudio en que la mayor extracción de nutrientes por el fruto siguen el orden de $\mathrm{K}>\mathrm{N}>\mathrm{Ca}$.Diagnóstico nutricional. En el diagnóstico inicial, DRIS y DOP, para la etapa 1 coincidieron en la clasificación de $\mathrm{K}$ como deficiente y $\mathrm{P}, \mathrm{Ca}$ y $\mathrm{Mg}$ como en exceso, mientras que la clasificación de $\mathrm{N}$ y $\mathrm{S}$ varió entre métodos. En la etapa 2 coincidieron en la clasificación de los nutrientes $\mathrm{N}, \mathrm{K}$ y $\mathrm{Ca}$; en las etapas 3 y 4 coincidieron en todos los nutrientes excepto $\mathrm{Mg}$, encontrándose $\mathrm{N}, \mathrm{P}, \mathrm{S}$ en el rango de deficiente, $\mathrm{y}$ $\mathrm{K}$ y $\mathrm{Ca}$ en el rango de excesivos (Cuadro 7).

$\mathrm{Al}$ determinar el orden de requerimiento nutricional para la siguiente cosecha a partir de la mayor deficiencia se encuentra que por el método DRIS el orden es $\mathrm{Mn}>\mathrm{Cu}>\mathrm{Fe}>\mathrm{N}>\mathrm{P}>\mathrm{S}$ y por el método DOP $\mathrm{Cu}>\mathrm{N}>\mathrm{S}>\mathrm{Mg}>\mathrm{Fe}>\mathrm{P}$. Los métodos coinciden en cinco de los nutrientes deficitarios, sin embargo, el orden de deficiencia cambia. El índice de balance nutricional (IBN) calculado para el método DRIS determinó que existió un desequilibrio nutricional alto en la etapa 1, pero 
decreció a medida que se avanzó en el desarrollo del cultivo, teniendo en cuenta que cuanto mayor es el IBN, mayor es el desequilibrio nutricional (Beaufils, 1973; Mourão, 2003).

Cuadro 7. Diagnóstico nutricional basado en las metodologías DRIS y DOP en cuatro etapas fenológicas del mango cv. Azúcar en el departamento del Magdalena*

\begin{tabular}{|c|c|c|c|c|c|c|c|c|c|c|c|c|}
\hline \multicolumn{13}{|c|}{ Índice DRIS } \\
\hline & $\mathrm{N}$ & $\mathrm{P}$ & K & $\mathrm{Ca}$ & $\mathrm{Mg}$ & $S$ & $\mathrm{Fe}$ & $\mathrm{Cu}$ & $\mathrm{Mn}$ & $\mathrm{Zn}$ & B & IBN \\
\hline Etapa 1 & 11,8 & 14,72 & $-52,21$ & 9,68 & 14 & 16,49 & $-87,16$ & 279,77 & $-90,68$ & $-18,81$ & $-83,12$ & 678,44 \\
\hline Etapa 2 & $-2,39$ & 1,13 & 5,15 & $-6,54$ & 1,68 & 0,98 & $-56,62$ & 58,21 & $-26,61$ & $-26,47$ & $-2,83$ & 188,61 \\
\hline Etapa 3 & $-1,77$ & $-6,15$ & 2,09 & 7,71 & $-1,21$ & $-0,68$ & $-25,78$ & 22,58 & $-20,34$ & $-51,97$ & 24,24 & 164,52 \\
\hline Etapa 4 & $-5,45$ & $-2,4$ & 3,63 & 3,39 & 2,29 & $-2,08$ & $-8,87$ & $-18,21$ & $-21,27$ & 24,47 & 23,89 & 115,95 \\
\hline \multicolumn{13}{|c|}{ Índice DOP } \\
\hline & $\mathrm{N}$ & $P$ & K & $\mathrm{Ca}$ & $\mathrm{Mg}$ & $S$ & $\mathrm{Fe}$ & $\mathrm{Cu}$ & $\mathrm{Mn}$ & $\mathrm{Zn}$ & B & \\
\hline Etapa 1 & $-4,44$ & 1,29 & $-27,28$ & 3,44 & 6,34 & $-0,14$ & $-6,49$ & $-16,93$ & 4,19 & 8,21 & 12,16 & \\
\hline Etapa 2 & $-4,5$ & $-0,29$ & 5,02 & $-3,47$ & $-2,95$ & $-1,25$ & $-12,88$ & $-26,15$ & 11,48 & $-0,04$ & 31,24 & \\
\hline Etapa 3 & $-4,09$ & $-8,5$ & 0,52 & 8,34 & 0,75 & $-0,38$ & 2,17 & $-19,22$ & 16,24 & 6,8 & 32,46 & \\
\hline Etapa 4 & $-4,22$ & $-0,3$ & 5,72 & 7,45 & $-0,54$ & $-0,77$ & $-0,48$ & $-10,45$ & 15,95 & 7,53 & 30,34 & \\
\hline
\end{tabular}

* Valores negativos indican deficiencia y valores positivos, exceso. IBN: índice de balance nutricional

\section{CONCLUSIONES}

La mayor extracción de nutrientes se presenta en los primeros 40 días de formación de la fruta para la mayoría de los nutrientes, siendo la mayor extracción por tonelada de fruta de $\mathrm{K}$ seguido de $\mathrm{N}$. De los microelementos la mayor extracción fue de Fe, seguido de B.

El orden de requerimiento nutricional determinado por los métodos DRIS fue $\mathrm{Mn}>\mathrm{Cu}>\mathrm{Fe}>\mathrm{N}>\mathrm{P}>\mathrm{S}$ y por el método DOP $\mathrm{Cu}>\mathrm{N}>\mathrm{S}>\mathrm{Mg}>\mathrm{Fe}>\mathrm{P}$. Por su parte, la productividad presentó correlaciones significativas con las concentraciones a nivel foliar de $\mathrm{P}, \mathrm{K}$ y $\mathrm{Mg}$.

Las concentraciones de nutrientes a nivel foliar obtenidas en los árboles de alta productividad pueden servir como referencia a estándares generales para diagnosticar el estado nutricional del cultivar mango cv. Azúcar establecido bajo condiciones edafoclimáticas del departamento del Magdalena, Colombia.

\section{AGRADECIMIENTOS}

Esta investigación fue realizada por la Corporación Colombiana de Investigación Agropecuaria-AGROSAVIA, con recursos recibidos en el marco de la ley 1731 de 2014. Los resultados son parte del proyecto "Estrategias de manejo del riego y la nutrición en periodos críticos de sequía para la producción sostenible de mango en zonas productoras de Colombia". Los autores expresan su agradecimiento a los productores Luis Mario Alzamora y Wilmar Garnica por el apoyo brindado en la ejecución del proyecto y por permitir el ingreso a sus fincas para el montaje del experimento.

\section{LITERATURA CITADA}

1. Agronet, 2020. Evaluaciones Agropecuarias Municipales 2013. Grupo de estadística e información sectorial - Oficina de Planeación y Prospectiva - Ministerio de Agricultura y Desarrollo Rural. Bogotá, Colombia. http://www. agronet. gov. Co / estadística (consulta de enero 15, 2021).

2. Ahmad, I., F. Bibi., A. Bakhsh., H. Ullah, S. Danish. y Asif-ur-Rehman. 2018. Assessment of various levels of potassium citrate and sucrose along with boric acid on quality and yield of Sufaid Chaunsa, Intern. J. Biosci.13(1): 188-195.

3. Anees, M., A. Ali, U. Shakoor, F. Ahmed, Z. Hasnain. y A. Hussain. 2016. Foliar applied potassium and zinc enhances growth and yield performance of maize under rainfed conditions. Int. J. Agric. Biol. 18: 1025-1032.

4. Beaufils, E.R. 1973. Diagnosis and 
recommendation integrated system (DRIS) a general scheme for experimentation and calibration based on principle developed from research in plant nutrition. Soil Sci. Bui. No.1, Univ. of Natal, South Africa.

5. Beberly, R., J. Stara, J. Ojala y T. Embleton. 1984. Nutrient diagnosis of "Valencia" oranges by DRIS. J. Am. Soc. Hortic. Sci. 109: 649-654.

6. Bekele, M., N. Satheesh y J.A. Sadik. 2020. Screening of Ethiopian mango cultivars for suitability for preparing jam and determination of pectin, sugar, and acid effects on physicochemical and sensory properties of mango jam. Scientific African 7(5): e00277.

7. Bibi, F., I. Ahmad, A. Bakhsh., S. Kiran, S. Danish., H. Ullah y Asif-Ur-Rehman. 2019. Effect of foliar application of boron with calcium and potassium on quality and yield of mango cv. Summer Bahisht (SB). Chaunsa. Open Agriculture 4: 98-106.

8. Campos, B y E. Calderón. 2016. El Análisis Foliar para el Diagnóstico Nutritivo de Plantaciones de Mango. Toma de muestras. Málaga. Consejería de Agricultura, Pesca y Desarrollo Rural, Instituto de Investigación y Formación Agraria y Pesquera, 1-8 p. Formato digital (e-book) - (Producción Agraria)

9. Cruz, V., R. Bugarín., G. Alejo., G. Luna y P. Juárez. 2014. Extracción y requerimiento de macronutrimentos en mango 'Ataulfo' (Mangifera indica L.) con manejo de poda anual y bianual. Revista Mexicana de Ciencias Agrícolas 5(2): 229-239.

10.Dar, M.A., J.A. Wani., S. Raina., M.Y. Bhat y M.A. Malik. 2015. Relationship of leaf nutrient content with fruit yield and quality of pear. Journal of Environmental Biology 36: 649-653.

11.Fallas, R., F. Bertsch., M. Miranda y C. Henríquez. 2010. Análisis de crecimiento y absorción de nutrimentos de frutos de mango, cultivares Tommy Atkins Y Keith. Agronomía Costarricense 34(1): 1-15.

12.Faria, L., S. Donato., M. Dos Santos y L. Castro. 2016. Nutrient contents in "Tommy Atkins" mango leaves at flowering and fruiting stages. Eng. Agric. 36(6): 1073-1085.

13.Galán-Saúco, V. 2013. Worldwide mango production and market: current situation and future prospects. Acta Hortic. 992: 37-48.

14.Li, R.F., Y. Chang., T. Hu., X. Jiang., G. Liang, Z, Lu., Y. Yi y Q. Guo, 2017. Effects of different fertilization treatments on soil, leaf nutrient and fruit quality of Citrus grandis var. longanyou. World Journal of Engineering and Technology 5: 1-14.

15.Lucena, J. J. 1997. Methods of diagnosis of mineral nutrition of plants a critical review. Acta Hortic. 448: 179-192.

16. Malavolta. E. 2006. Manual de Nutrição Mineral de Plantas. Livroceres, São Paulo, $638 \mathrm{p}$.

17.Medeiros. A., J. Amorim., D. Silva., J. Dantas y A. Guerra. 2004. Mineral composition of leaves and fruits of irrigated mango trees in Rio Grande do Norte State, Brazil. Acta Horticulturae 645: 403-410.

18.Mellado-Vázquez, A., S. Salazar, R. Goenaga y A. López. 2020. Survey of fruit nutrient removal by mango (Mangifera indica L.) cultivars for the export market in various producing regions of Mexico. Terra Latinoamericana 37: 437-447.

19.Montañés, L., L. Heras, J. Abadía y M. Sanz.1993. Plant analysis interpretation based on a new index: Deviation from optimun percentage (DOP). J. Plant Nutr. 16: 1289-1308.

20.Mourão F.A. y J. Azevedo. 2003. DRIS norms for 'Valencia' sweet orange on three rootstocks. Pesq. Agropec. Bras. 38: 85-93.

21.Mourão, F.A. 2004. DRIS: concepts and applications on nutritional diagnosis in fruit crops. Sci. Agric. (Piracicaba, Braz.) 61(5): 550-560.

22.Nadir, M. 1972. Matieres fraiches, matieres seches et teneurs des elements mineraux dans les differents organes et parties des citrus en production. Al-Awamia 43: 17-30.

23. Obreza, T. y Morgan. K. 2008. Nutrition of Florida citrus trees. Gainesville: Soil and Water Science Department, University of Florida.

24. Osorio, 2014. Manejo de Nutrientes en Suelos del Trópico. Editorial L.Vieco. Medellín, Colombia. $412 \mathrm{p}$.

25.Puentes, Y., J. Menjivar y F. Aranzazu. 2016. Concentración de nutrientes en hojas, una 
herramienta para el diagnóstico nutricional en cacao. Universidad de Costa Rica. Agronomía Mesoamericana 27(2): 329-336.

26.Ranade-Malvi, U. 2011. Interaction of micronutrients with major nutrients with special reference to potassium. Karnataka Journal of Agricultural Sciences 24(1): 106-109.

27.Rodríguez, E., J. Gutiérrez y Orduz. J. 2018. Diagnóstico nutricional del cultivo de la lima ácida 'Tahití' [Citrus latifolia (Yu Tanaka) Tanaka] en el departamento del Tolima (Colombia). Temas Agrarios 23(2): 144-153.

28. Romero, I., Benito, A., Domínguez, N., GarciaEscudero, E. y I. Martin. 2014. Leaf blade and petiole nutritional diagnosis for Vitis vinifera $\mathrm{L}$. cv. 'Tempranillo' by deviation from optimum percentage method. Spanish Journal of Agricultural Research 12(1): 206-21.

29.Salehin, S. K., G. Mohsin, T. Ferdous, J. Zaman, Md. J. Uddin y Md. K. Rahman. 2020. Soil fertility and leaf nutrient status of mango orchard sites. Dhaka Univ. J. Biol. Sci. 29(2): 155-163

30.Sandoval, J., O. Galindo, A. Arellano y L. Ancira. 2019. Preliminary study of the fertirriego in the culture of the watermelon (Citrullus lanatus, Thumb) in Tolimán, Jalisco. Revista Iberoamericana de las Ciencias Biológicas y Agropecuarias 10(19).

31.Shaban, A., R. El-Motaium, S. Badawy y A. Ibrahim. 2019. Response of mango tree nutritional status and biochemical constituents to boron and nitrogen fertilization, Journal of Plant Nutrition 42(20): 2784-2793.

32.Souri M. K. y M. Bakhtiarizade 2019. Biostimulation effects of Rosemary essential oil on growth and nutrient uptake of tomato seedlings. Sci Hortic 243: 472-476.

33.Souri, M. K. y M. Aslani. 2018. Beneficial effects of foliar application of organic chelate fertilizers on French bean production under field conditions in a calcareous soil. Adv. Hortic. Sci. 32(2): 265-272.

34. Teixeira, T., I. Lucena, A. Nasciment, Y. Carvalho, P. Rodrigues y J. Gomes. 2019. Biostimulants on nutritional status and fruit production of mango 'Kent' in the Brazilian Semiarid Region. HortScience 54(9): 1501-1508.

35. Tohidloo G., M. Souri, S. Eskandarpour. 2018. Growth and fruit biochemical characteristics of three strawberry genotypes under different potassium concentrations of nutrient solution. Open Agri. 3: 356-362.

36. Walworth, J. L., W. S. Letzsch y M. E. Sumner. 1986. User of boundary lines in establishing diagnostic norms. Soil Sci. Soc. Am. J. 50: 123128.

37.Zekri, M., T. Obreza y A. Schumann. 2005. Increasing efficiency and reducing costs of citrus nutritional programs. Gainesville: Soil and Water Science Department, University of Florida. Sheet SL222. 6 p. 
